\title{
Does the Expansion of Higher Education Increase the Equality of Educational Opportunities? Evidence from Italy
}

\author{
Massimiliano Bratti - Daniele Checchi - \\ Guido de Blasio
}

Abstract. This paper studies the role of the expansion of higher education (HE) in increasing the equality of tertiary education opportunities. We examine Italy's experience during the 1990s, when policy changes prompted HE institutions to offer a wider range of degrees and to open new sites in neighbouring provinces. Our analysis focuses on non-mature full-time students and suggests that HE expansion might have had only limited effects in terms of reducing existing individual

Massimiliano Bratti (author for correspondence) - Daniele Checchi_ Guido de Blasio, Dipartimento di Scienze Economiche Aziendali e Statistiche (DEAS), Università degli Studi di Milano, via Conservatorio 7, I-20122 Milan, Italy. E-mail: massimiliano.bratti@unimi.it.

This project was developed in part while Massimiliano Bratti was visiting the Institute for the Study of Labor (IZA, Bonn, October 2006) and the European Centre for Analysis in the Social Sciences (ECASS, Institute for Social and Economic Research, University of Essex, April-May 2007). He wishes to thank both institutions for providing him with excellent research facilities. The visit to ECASS was financially supported by the Access to Research Infrastructure action under the European Community's 'Improving Human Potential Programme', which is gratefully acknowledged. We thank Giuliana Matteocci and Alessio Ancaiani from the Ministry for Education for providing us with data on the evolution of the Italian university system and for valuable assistance with its use. We are also grateful to an anonymous referee and to Luigi Cannari, Piero Casadio, Massimo Omiccioli, Carmine Porello, Andrea Presbitero, Alfonso Rosolia, Paolo Sestito, and conference and seminar participants at the Institute for Social and Economic Research (University of Essex, Colchester), the European University Institute (Florence), the University of Milan (Milan), the Marche Polytechnic University (Ancona), the Bank of Italy (Rome), the joint seminar ISFOL-University 'La Sapienza' (Rome), IZA (Bonn), the 2007 Conference of the Italian Association of Labour Economists (AIEL, Naples) and the 2007 Conference of the European Association of Labour Economists (EALE, Oslo) for comments and suggestions. We gratefully acknowledge Christine Stone's editorial assistance. The usual disclaimers apply.

LABOUR 22 (Special Issue) 53-88 (2008)

JEL 12

(C) 2008 The Authors

Journal compilation (C) 2008 CEIS, Fondazione Giacomo Brodolini and Blackwell Publishing Ltd., 9600 Garsington Rd., Oxford OX4 2DQ, UK and 350 Main St., Malden, MA 02148, USA. 
inequality in HE achievement as it had a significantly positive impact only on the probability of university enrolment but not on that of obtaining a university degree.

\section{Introduction}

This paper studies the impact of the expansion in the supply of higher education (HE hereafter) on equality of opportunity in individual educational careers at tertiary level. Despite the existence of alternative definitions of equality of opportunity (Roemer, 1998), we content ourselves with the notion of increasing equality of opportunity associated with a reduction in the influence of events that are outside individual control (e.g. what Roemer defines as circumstances). Educational choices are typically correlated with parental features, including role models, education, cultural resources, current income, residential choices, and inherited wealth (Carneiro and Heckman, 2003). ${ }^{1}$ Thus, any policy capable of reducing the impact of one (or more) of these variables on individual educational choices can be regarded as reducing inequality (of opportunity). A well-known example is compulsory education laws. As each child is required to attend school irrespective of his/her family of origin, this obligation reduces (and in the extreme case of full compliance eliminates) the differential in the probability of students from different family backgrounds completing a given level of education. The other side of the coin is that an increase in equality of opportunity translates into an increase in intergenerational mobility in education whenever parental education is the crucial variable characterizing family background (Machin and Blanden, 2004).

In this paper we focus on a specific segment of the educational career, i.e. access to and completion of tertiary education. Although secondary education is almost universally achieved in most developed countries, tertiary education still represents a discriminating factor between students from different social classes (Shavit et al., 2007). Most countries have accommodated the increasing demand for $\mathrm{HE}$ by introducing non-university tertiary education (technical schools, polytechnics, fachhochschulen), whereas others (including Italy) have retained a university-based organization of tertiary education. As a consequence, existing institutions have faced mounting pressure to accommodate a student population that has decupled in Italy over the last three decades. The new entrants were the offspring of less privileged families, thus increasing equality of opportunity almost by definition. However, what matters is the odds ratio between two otherwise identical individuals born in different 
families, and it is not obvious that an expansion of HE especially benefits individuals from worse family backgrounds (see Machin and Blanden, 2004). It is crucial to analyse how the enlargement of university access has been accomplished. One could increase the number of places available, introducing in the meanwhile a screening of students based on their academic abilities. Conversely, one could open tertiary education to the private sector, allowing the creation of an implicit ranking of existing institutions and leaving the market for $\mathrm{HE}$ to achieve some equilibrium configuration (Fernandez and Gali, 1999).

The case of Italy is an interesting one in several respects. Italian policy makers did not pursue the route of stratifying the supply of tertiary education (between university and non-university or between public and private), but followed the principle of autonomy, encouraging local solutions while still retaining a regime of central approval. In terms of equality of opportunity this choice may have ambiguous effects. On one side, the generalized expansion of enrolment may increase equality. On the other side, locally devised solutions may exacerbate poverty traps (either economic or cultural), thereby reducing equality. Things are even more complicated when we consider that there were a number of key changes in the regulation of the university system in Italy in the 1990s (see Appendix A). As a result, Italian universities started to expand their supply, both by opening new sites in neighbouring cities and by offering a broader variety of degrees. Students and their families were confronted with a changing and expanding supply, which is likely to produce at least two effects: a cost-reduction effect, associated with the possibility of enrolling at university without moving to a different city; and a potential increase in labour market returns, given the increased possibility of selecting courses that are better tailored to the needs of employers. We are not surprised to observe, therefore, that the increased supply of HE went hand in hand with expanding demand. However, we are more interested in understanding which students benefited most from this expansion. The regional expansion of HE supply is the source of variability that we exploit to understand the relationship between the availability of university courses and infrastructures at the local level and the likelihood of attending and/or possibly completing a university course.

The macro-evidence is unable to provide a reliable answer to this question. Although university statistics show a sharp increase in student and graduate numbers in Italy during the 1990s, it is not clear whether this rise was determined primarily by changes in the 
characteristics of the population (e.g. the number of individuals with highly educated parents) and/or in the macroeconomic environment (e.g. an increase in the returns to education due to skill-biased technical change) or whether the increase in HE supply that took place in the same period instead had an independent effect on the demand for education. On the contrary, using evidence from micro-data, we show that the expansion of university courses has effectively increased the likelihood of university enrolment for students from middle-class and/or less educated parents. However, the expansion in enrolment has not translated into an increased probability of attaining a degree. Thus, we observe a reduction in inequality of opportunity that is only apparent, as the odds of students from different social backgrounds graduating seem unaffected by the increased availability of university courses.

The paper is organized as follows. Section 2 briefly describes the policy changes introduced in the 1990s. Section 3 illustrates our empirical strategy and the main results and provides some possible explanations. Section 4 concludes.

\section{1990-2000: a decade of expansion of HE in Italy}

At the end of the 1980s, the public system of HE in Italy was in a dismal state (see ISTAT, 1995). The graduation rate was one of the lowest of the Organization for Economic Cooperation and Development (OECD) countries, as only 30 per cent of the students enrolled actually managed to attain a degree. The (median) time span required to complete a degree was twice that envisaged. The university poles of the largest metropolitan areas were overcrowded.

It was widely believed that the failure of the public system of HE could be tackled. Whereas before the 1990s the main feature of Italy's university system had been its substantial immobility, during that decade there was a rush of policy interventions, which are detailed in Appendix A. For the purpose of our paper it is worth noting that these reforms resulted in a spectacular increase in the supply of HE. First, a law was passed forcing the largest universities (Milan, Rome and Naples, which at that time had more than 40,000 students) to split up, and this triggered the birth of a number of smaller sites. Second, new public funding was granted to expand HE infrastructures, especially in the South, which had fewer university premises. Third, and crucially, the Italian Parliament passed a series of laws giving Italian universities substantial, and entirely new, autonomy. 
Given the soft budget constraint, this autonomy prompted the universities to adopt measures to expand their HE supply.

As Besley and Case (2000) point out care should be taken when drawing inference from policy variations, which might be sensitive to economic conditions (endogenous policies). The increase in HE supply was not driven by an economic motive: the new infrastructures did not follow the potential unfilled demand for HE, and cost-benefit analyses were never performed. Rather, the increase followed an indiscriminate allocation of public funds across the Italian regions. Consider the following assessment taken from a document published by the Italian Ministry of Education, University and Research (MIUR, 1997, p. 10 - our translation):

In respect of the development and rebalancing of university educational supply, which had to be carried out on a regional basis, a large number of actions were carried out: 4 new universities were founded, 2 private universities became state universities, 17 new sites were created, 41 new faculties were instituted and no less than 230 new degrees were created (adding to the 890 existing in 1986). Nevertheless, (...) these actions were not planned taking into account the educational demand expressed by each region, or evaluating the potential flow of students (i.e. evaluating the potential demand for each action), the employment perspectives (i.e. the competences and skills required by the country) or the potential of existing facilities. Ultimately, these actions lacked accurate assessments regarding either their comprehensive scope or their compatibility with the pre-existing situation. In fact, the main purpose of the programmes seemed to be a geographical rebalancing of universities premises in order to bring the supply of education closer into line with the demand, whereas issues such as the real extent of that demand (which was sometimes so small as to make an efficient and effective endeavour impossible) and the situation regarding infrastructures, accommodation and financial support available to students were disregarded. Once again an indiscriminate and illdirected approach prevailed, inspired by a barely incremental purpose...

The 'quasi-randomness' of HE expansion with respect to potential demand is key to our identifying strategy. In fact, we aim to establish whether larger HE supply created larger HE demand, i.e. causation must run from supply to demand. For this reason, we must be sure that supply was not affected by factors influencing 
Table 1. Evolution of the HE supply in Italy

\begin{tabular}{lrrrr}
\hline & $\mathbf{1 9 8 5}$ & $\mathbf{1 9 9 0}$ & $\mathbf{1 9 9 5}$ & $\mathbf{2 0 0 0}$ \\
\hline Universities & 55 & 58 & 60 & 70 \\
Cities with a university & 42 & 42 & 45 & 50 \\
$\quad$ head office & & & & \\
Cities with a university site & 47 & 62 & 93 & 146 \\
Schools (facoltà) & 329 & 365 & 412 & 474 \\
4- to 6-year degrees & 778 & 898 & 988 & 1,321 \\
2- to 3-year degrees & - & - & 388 & 956 \\
\hline
\end{tabular}

Source: CNVSU, La localizzazione geografica degli atenei statali e non statali in Italia dal 1980 al 2000, 2001.

both supply and demand or by potential demand, which we might have omitted from our econometric models.

Universities pursued different strategies to expand supply. Apart from those who were forced by law to split, some universities just opened subsidiaries (e.g. Bologna in Forlì and Rimini, Siena in Arezzo), whereas others preferred to open multicentre universities (e.g. Piemonte Orientale, Insubria, Modena, and Reggio Emilia). ${ }^{2}$ Moreover, some universities replicated their existing supply across the country (e.g. the Catholic University opened short courses in nursing in 10 different locations), whereas others diversified into different fields (e.g. the universities of Salerno, Cassino, and Benevento opened four new faculties each). ${ }^{3}$

As a result, the variety of degrees boomed whereas the geographic concentration of $\mathrm{HE}$ infrastructures decreased sharply. On the one hand, the total number of 4- and 5-year degrees (corsi di laurea) on offer rose from 898 to $1,321 .{ }^{4}$ On the other hand, universities - historically concentrated in the largest cities expanded their supply to small cities. From 1990 to 2000, the number of towns with a university site rose from 104 to 196 . The data reported in Table 1 show that this expansion took place mainly between 1990 and 2000. The territorial distributions of the expansion in the number of courses and sites, normalized by the population aged 19, are depicted in Figures 1 and 2, respectively. These two measures, courses and sites, are used to proxy the expansion of HE supply in the empirical section below. Our main indicator, new courses, is the average yearly increase in the number of courses (divided by the local population aged 19 in 1991) at the regional level (Figure 1). This variable reflects both the opening of new courses, possibly but not necessarily in different fields, in sites 
Figure 1. Expansion of university supply: yearly number of new courses introduced during $1990-2000$ by region per 1,000 inhabitants aged 19

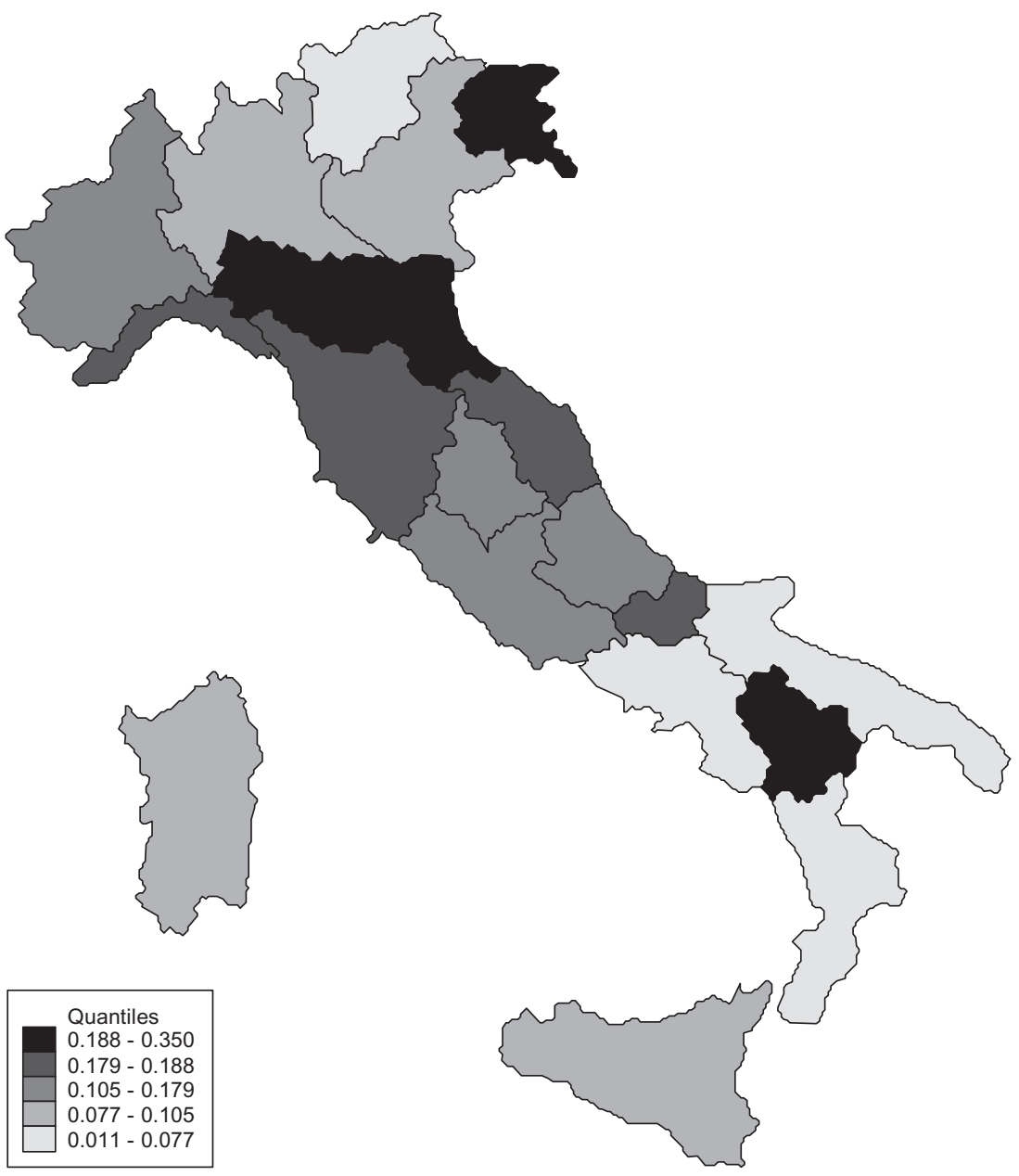

already served by university infrastructures and courses in sites not previously endowed with HE infrastructures. The impact of this variable can therefore reflect both 'variety' benefits (due to the availability of courses with up-to-date contents and curricula better tailored to local labour market needs) and 'proximity' benefits (which materialize if the local availability of infrastructures reduces attendance costs). In an attempt to disentangle these two potential 
Figure 2. Expansion of university supply: yearly number of new locations created during $1990-2000$ by region per 1,000 inhabitants aged 19

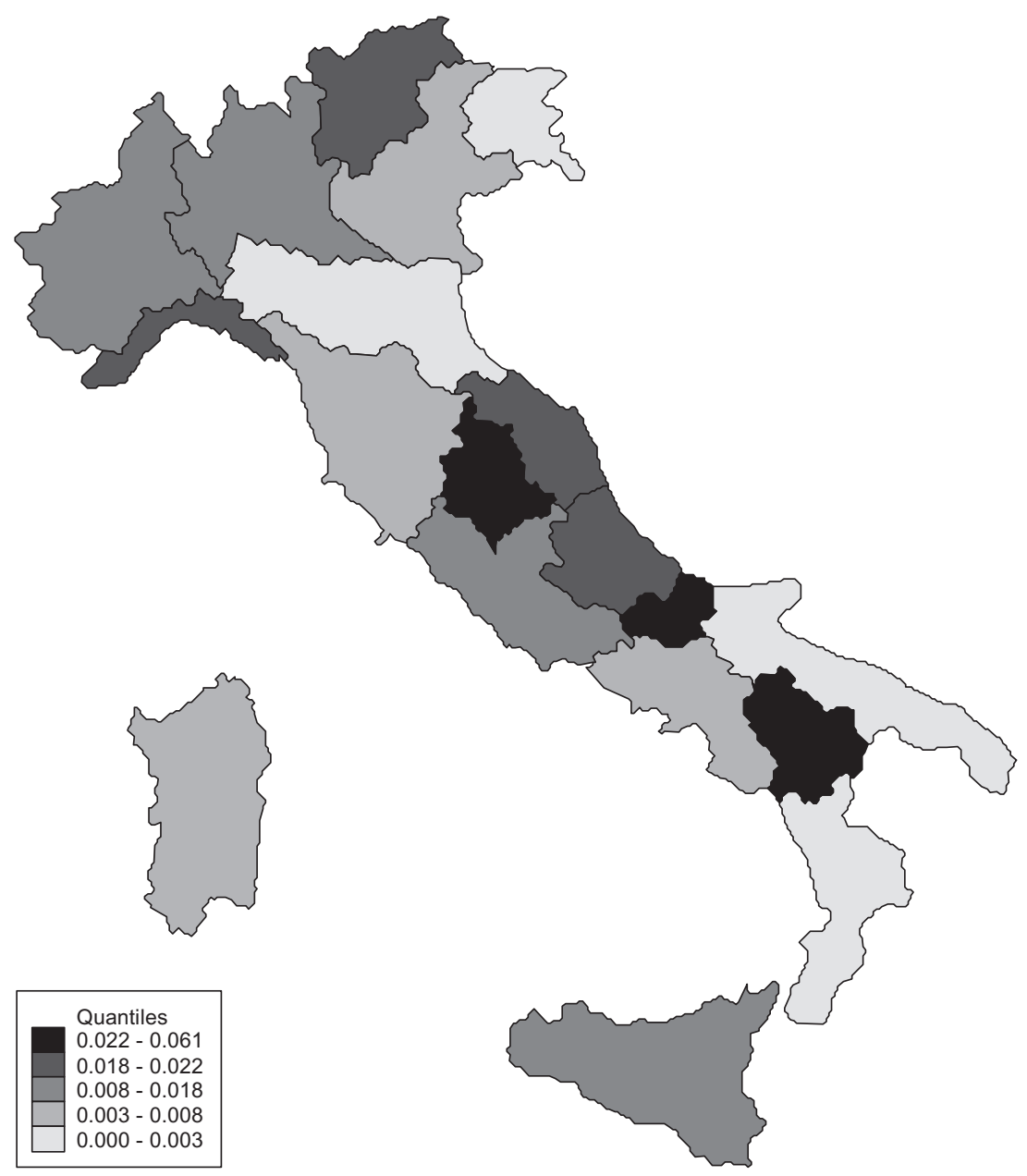

sources of benefits, we also make use of another indicator, new sites, which is the average yearly increase in the number of university locations (divided by the total population aged 19 in 1991) at the regional level (Figure 2). Although some overlap remains (e.g. the content of the courses created in new sites was sometimes different from that of the courses supplied in the established sites), this second indicator should reflect more accurately the role of the 
increased diffusion of $\mathrm{HE}$ infrastructures over the region. By comparing the two maps it is easy to see that sufficient variability exists at local level in terms of alternative expansion strategies.

\section{Empirical strategy and results}

The analysis in this section seeks to answer the following question: do the regions that expanded HE supply most also have ceteris paribus a higher fraction of graduate population? As will be made clearer later on, we focus on the population aged 23-31, considering the fraction of population enrolled full-time at university or already possessing a university degree. As these individuals were potentially exposed to the expansion of HE supply, we wonder whether this fraction is larger in regions that expanded HE most. In addition, we also ask which individuals benefited most from this expansion in the supply of HE. And last but not least, we ask whether these correlations reflect causal effects.

To answer these questions we exploit the increase in the supply of HE promoted by the reforms of the 1990s to evaluate its effects on the equality of tertiary education opportunities. We use some crosssections of individuals for whom we have information from the Bank of Italy's Survey of Household Income and Wealth (SHIW) and we link individual educational attainment with region-level data on the magnitude of the increase in regional HE supply between 1990 and 2000. The 'exposure' of an individual to the 'treatment' (i.e. HE supply expansion) and its intensity are determined by the period in which he/she attended/did not attend university (cohort of birth) and by his/her region of residence, respectively. ${ }^{5}$ Although exposure to the post-1990s educational reforms is completely exogenous (depending only on an individual's birth cohort), the intensity of the treatment is potentially endogenous. For this reason we investigate the extent to which our results change by considering potential omitted variables that might be simultaneously correlated with both the demand and the supply of tertiary education. Basically, we calculate the probability for an individual with a given family background to achieve a university degree and assess whether this probability (which depends on family background) has changed because of the expansion of HE supply.

One limitation of the SHIW data is that we observe only the region of birth and the current region of residence, whereas we do not observe the region of residence at the relevant age (typically 19 
years old) when decisions about enrolling at university are usually made. We are therefore forced to assume that the current region of residence was also the region of residence at age 19, or whenever an individual took the decision whether to enrol at university or not. Note that in the case of Italy this assumption seems quite reasonable. The inter-regional mobility of university students is low: more than 80 per cent enrol at a university located in the same region where they reside, and this share has been roughly constant over the decade (see MIUR, 2003). Moreover, the vast majority of Italian graduates live and work in the same province where they studied. ${ }^{6}$

Nevertheless, we do not take this assumption for granted and investigate the implications of its failure. Our main concern is the possibility of endogenous migration. For instance, some individuals might move to regions where HE expansion is greater simply because they want to have a wider choice for HE enrolment and then find a job in the same region. ${ }^{7}$ In addition, university-educated individuals who obtained their degrees elsewhere may be attracted by high HE supply regions in the expectation of an expanding labour market for graduates. In both cases, observing a positive correlation between HE supply expansion and tertiary educational achievement measured at regional level would only reflect a spurious one. ${ }^{8}$ The bias need not be positive. Some individuals from better family backgrounds may react to the rapidly increasing local HE supply by choosing traditional and well-reputed institutions and courses rather than new local ones. They might even move to other regions, in which case we would observe a negative correlation between HE expansion and educational attainment of individuals from better backgrounds, as the ones still observed in the regions that expanded HE are those who discarded university enrolment. The potential consequences of endogenous migrations for our estimates are analysed in Section 3.2.

We consider two different indicators of educational achievement: (1) the likelihood of holding a university bachelor degree; and (2) the likelihood of holding a bachelor degree or of being a full-time university student. ${ }^{9}$ The former measure (holding a university degree) takes into account not only enrolment but also the effectiveness of the educational process, i.e. whether (and when) a degree is obtained. For instance, an increase in student enrolments due to HE expansion would be considered a positive outcome if and only if these students completed their studies before age 31, which is the upper age limit for the sample we consider below (see Section 3.1). ${ }^{10}$ A well-known problem with Italian HE students is that the number 
of those who complete HE later than the envisaged completion time (fuori corso, beyond prescribed time) is huge. To the extent that supply expansion lowers the actual completion time, this improvement will be reflected in the first outcome variable. ${ }^{11}$ However, if the expansion of HE supply raises the enrolment probability of less motivated or less able students ('marginal students'), which typically have longer graduation times, these outcomes would not be captured by the former measure. This is why we also consider the second educational outcome variable.

A second limitation of the SHIW is that it identifies only those students who self-declared studying as their main activity (i.e. fulltime students). As a consequence, our data do not fully capture working students (i.e. part-time students) or mature students (who are less likely to self-define as students). This means that in the analysis that follows we are not able to evaluate fully the effect of $\mathrm{HE}$ expansion on mature students as they are likely to be full-time or part-time workers and to graduate when they are over 31 . However, as both pecuniary and non-pecuniary returns of a university degree (increase in productivity and wage, healthier attitudes) are greater for younger individuals because their lifetime horizon is longer, we think that combining both measures of education may provide a balanced view of the potential gain from HE expansion. ${ }^{12}$

As often happens in empirical research we do not have an ideal data set for our research goals. For a proper identification of the effects of supply expansion, we would like to compare individuals exposed to the increase in HE supply with fully unexposed ones. At the same time, we would also like to control for any other possible determinant of tertiary education. Our data do not enable us to achieve both objectives at once because we do not possess all the relevant information about the external circumstances when the individual took the crucial decision about HE enrolment. We amend these deficiencies by taking into account various outcome variables and by resorting to a 'quasi-experimental' design in data analysis. Although each has its pros and cons, we maintain that overall they provide a general view of the effectiveness of expansion of HE supply.

\subsection{The baseline specification}

The SHIW does not generally provide individuals' graduation date. To contrast exposed individuals with unexposed ones we have to select carefully the relevant cohorts from different SHIW waves. 
Let us start by considering a baseline case. In this first analysis we use the 1993 and the 2002 waves of the SHIW and consider individuals aged 23-31 in both waves. All individuals who were 23-31 in 1993 and held a degree must have enrolled in HE before the expansion (the 'treatment') that took off at the beginning of the 1990s (see Table 1). These individuals are considered to be untreated. We select individuals in the same age group from the 2002 wave. As the regular entry age into HE in Italy is 19 (20 for one-third who repeated at least 1 year), all individuals who held a degree in 2002 and were aged 23-31 in the same year must have enrolled at university in 1990 or later, i.e. during the years of expanding supply. ${ }^{13}$

To assess the effect of the expansion of university supply on individual educational outcomes we not only compare the educational outcomes of individuals with similar characteristics before and after the 1990s reforms (comparing two cross-sections), we also consider the effect of differences in local intensities of treatment (HE expansion). ${ }^{14}$ Although birth cohort determines treatment status, treatment intensity is determined by the size of supply expansion measured at regional level.

However, the variation in treatment intensity across regions and cohorts can be meaningfully linked to individual human capital attainments only if we are able to differentiate out individual and regional characteristics that may be correlated with the supply expansion and may also affect the decision to enrol in HE (i.e. demand). Previous work suggests that family background is generally a powerful predictor of an individual's educational outcome in Italy (Checchi et al., 2007). Controlling for parental background is therefore central to identifying correctly the causal effect of the expansion in university supply, as one could object that the accumulation of human capital has grown simply due to the increase in the number of university-educated parents following a secular upward trend. Indeed, as university-educated parents are better able to afford $\mathrm{HE}$ costs for their offspring, and also attribute particular value to their children's education, we would observe an increase in HE enrolment that would be independent of existing supply conditions. ${ }^{15}$ A central feature of the 1993 and 2002 SHIWs used in the baseline specification is that they report information on parental education and job qualification. ${ }^{16}$ In the empirical specification we also include other individual characteristics such as gender and age, birth cohort, and region of residence.

Apart from the limitations of the data, the validity of our estimates relies on the identifying assumption that there are no omitted 
time-varying region-specific effects correlated with both HE expansion and individual educational achievement (see Borland et al., 2005, p. 17). This assumption cannot be taken for granted as the expansion in university supply during the 1990s could be correlated with other contemporaneous regional changes, which might have affected the demand for education. For instance, an increase of unemployment rates at regional level may produce an increase in the demand for HE (due to lower opportunity costs of acquiring human capital) and be positively correlated with HE expansion in the case of government compensatory policies. Similarly, an increase in real disposable income per capita may increase the demand for HE (either because it relaxes credit constraints or because it raises the demand for cultural consumption) and be positively correlated with the expansion of HE supply, as setting up new courses and opening new branches generally require external funding. ${ }^{17}$ Moreover, universities might expand their supply in response to the observed or expected rise in potential demand. During the 1990s Italian regions may have had differential rates of expansion of secondary education and demographic trends. If the supply expansion simply mimics the rise in the number of individuals completing upper secondary school, i.e. if universities open new branches or set up new courses in regions where the demand for education is currently rising (or expected to rise in the near future), then the estimated effect could hardly be considered to be supply driven.

For these reasons, we re-estimate the baseline specification also controlling for the absolute variation in unemployment rates (source: Prometeia), the growth rate in per capita disposable income at 1995 prices (source: Prometeia), and the change in the number of upper secondary school diploma-holders (source: Italian National Statistical Institute, ISTAT) and check the robustness of our results. All these additional control variables are measured between 1990 and 2000 at the regional level and are included as interactions with a post-reform dummy (which takes the value one for the post-reform cohorts and zero otherwise).

Following Duflo (2001) we specify the educational attainment function using a linear probability model (LPM). ${ }^{18}$ Let us define as EDUCATION a dichotomous variable representing the educational outcome of interest (e.g. either 'possessing a degree' or 'possessing a degree or being a full-time undergraduate student') defined at individual level ${ }^{19}$ that is assumed to be a linear function of some observable and unobservable characteristics and takes the value one if the educational outcome has been achieved: 


$$
\begin{aligned}
\text { EDUCATION }= & \mathrm{b}_{0}+\mathrm{b}_{1} \text { REGION }+\mathrm{b}_{2} \text { COHORT }+\mathrm{b}_{3} \text { BACKGROUND } \\
& +\mathrm{b}_{4} \text { POSTREFORM } * \text { BACKGROUND }+\mathrm{b}_{5} \text { POSTREFORM } \\
& * \text { BACKGROUND } * \Delta \text { SUPPLY }+\mathrm{b}_{6} \mathrm{X}+\mathrm{b}_{7} \text { REGIONVAR } \\
& * \text { POSTREFORM }+\varepsilon
\end{aligned}
$$

where REGION is a region of residence fixed effect, COHORT is a birth cohort fixed effect, BACKGROUND is a dummy variable measuring the individual family background (in terms of either parental education or occupation) ${ }^{20}$ POSTREFORM is a dummy variable that takes the value zero for all pre-reform cohorts and one for the postreform cohorts (i.e. the treatment status indicator), $\triangle$ SUPPLY is the expansion in university supply in the region of residence of the individual over the period 1990-2000, $\mathrm{x}$ is a vector of individual characteristics including gender, age, and the dimension of family background that is not interacted with the post-reform dummy and the increase in supply, REGIONVAR is a vector of regional control variables for the period 1990-2000, and $\varepsilon$ is a zero-mean stochastic error term that can be correlated within region and cohort (standard errors are clustered by region and birth cohort), capturing individual unobservable attributes. It is perhaps important to note that in specification [1] region fixed effects capture regional timeinvariant unobservables, cohort fixed effects capture country-level time trends in the demand for education, whereas the interaction between family background and the post-reform dummy allows these trends to differ by family background. The expansion of HE supply enters equation [1] interacted with the POSTREFORM dummy as only the post-1990s cohorts were exposed to it, and interacted with family background because we are interested in the differential effect of HE regional supply by family background captured by the $\mathrm{b}_{5}$ coefficients.

Given the relatively small sample size, we have considered alternative synthetic measures of family background. As to social class, three broadly defined social classes are used: working class or out of the labour force including blue collars, unemployed, or inactive; petite bourgeoisie including low-rank white collars, teachers, and self-employed workers; bourgeoisie including high-rank white collars, managers, members of the professions, and entrepreneurs. Alternatively, we have defined three levels of cultural capital: low when both parents have less than an upper secondary school diploma; medium when at least one parent has an upper secondary school diploma; high when at least one parent has a university degree. Sample descriptive statistics are reported in Table B1 in 
Appendix B and are not commented here. Each estimated regression includes either the interaction of HE supply with the social class or its interaction with the cultural capital. The importance of controlling for the heterogeneity of the effect of HE supply according to these two dimensions is justified by the fact that the first proxy of family background should capture mainly the effect of family 'economic capital' (in the absence of family income), whereas the second should proxy 'cultural resources'. In general, in Italy parents' education appears to be a much stronger predictor of children's education than parents' income (see, for instance, Checchi, 2003). The interactions between social class or cultural capital dummies and the post-reform dummy also help to capture the fact that students with the same social class or cultural capital in the pre- and post-1990 periods are likely to have very different levels of real income (due to economic growth) or pressures to acquire HE (e.g. in case of skill-biased technological change).

Table 2 reports the effect of the creation of new courses on the likelihood of holding a university degree. Section a of the table, reporting the estimated coefficients of the interactions with social class, does not show any significant effect of creation of new courses on the probability of holding a degree whether regional controls are omitted or included. In contrast, the interactions with cultural capital reported in section $b$ show a significant positive effect only for low-cultural capital individuals, although this is not robust to the inclusion of growth in per capita income. Therefore, including per capita income wipes out most of the effect of supply expansion. ${ }^{21}$ Curiously enough, our estimates also show a significant negative effect of creation of new courses on the probability of having a degree for individuals endowed with high cultural capital. Multiplying the coefficient on high cultural capital in the most complete specification of column (5) by the actual average increase in the number of courses in the 1990 s, which was 0.13 courses per year per 1000 individuals aged 19 , we obtain an effect of -21 percentage points. ${ }^{22}$ As this negative effect on high-background individuals is difficult to interpret and may be driven by endogenous migration, as we anticipated in the initial paragraphs of Section 3, we comment on it in Section 3.2 in an attempt to account for this potential problem.

Table 3 reports the effects of creation of new university sites. Here again only one effect remains robust to the inclusion of regional controls, and again it is negative and concerns highbackground individuals, namely those from the bourgeoisie. Multiplying the coefficient on the bourgeoisie in column (5) by the 
Table 2. Effect of increase in the number of university courses on the probability of holding a university degree

Effect of expansion

a. By social class

Working class or out of

labour force

Petite bourgeoisie

Bourgeoisie

$R$-squared

b. By cultural capital

Low

Medium

High

$R$-squared

Regional control variables

$\Delta$ Unemployment rate (1990-2000)

Growth rate per capita real disposable income (1990-2000)

$\Delta$ Secondary school graduates (1990-2000)

No. observations
(1)

(2)

(3)

(4)

(5)

Notes: The treatment is the average yearly increase in the number of university courses in the period 1990-2000 at regional level divided by the population aged 19 in 1991. Absolute value robust $z$ statistics in brackets (standard errors are clustered at region by cohort level); ${ }^{*}$ significant at 10 per cent; $* *$ significant at 5 per cent; $* * *$ significant at 1 per cent. Models also include cohort of birth dummies, age, region of residence dummies, gender, parental social class, parental cultural capital, and the interaction between parental social class and the post-reform dummy (section a) or the interaction between cultural capital and the post-reform dummy (section b). Observations are weighted to population proportions.

average yearly increase in the number of sites per 1000 inhabitants aged 19 during the 1990s (0.01), we obtain an effect of about -6 percentage points. By combining the results of Tables 2 and 3 we observe that the relative advantage of individuals from well-off families is reduced in terms of access to HE. We can therefore interpret this evidence as showing that the policy reform had an equality of opportunity-enhancing effect. An alternative reading of 
Table 3. Effect of increase in the number of university locations on the probability of holding a university degree

Effect of expansion

a. By social class

Working class or out of

labour force

Petite bourgeoisie

Bourgeoisie

$R$-squared

b. By cultural capital

Low

Medium

High

$R$-squared

Regional control variables

$\Delta$ Unemployment rate (1990-2000)

Growth rate per capita real disposable income (1990-2000)

$\Delta$ Secondary school graduates (1990-2000)

No. observations
(1)

(2)

(3)

(4)

Notes: The treatment is the average yearly increase in the number of university sites in the period 1990-2000 at regional level divided by the population aged 19 in 1991. Absolute value robust $z$ statistics in brackets (standard errors are clustered at region by cohort level); * significant at 10 per cent; ** significant at 5 per cent; *** significant at 1 per cent. Models also include cohort of birth dummies, age, region of residence dummies, gender, parental social class, parental cultural capital, and the interaction between parental social class and the post-reform dummy (section a) or the interaction between cultural capital and the post-reform dummy (section b). Observations are weighted to population proportions.

the same evidence indicates that the elites (defined in economic and/or cultural terms) were somehow damaged by the expansion of mass access to universities as they seem to have lost some of their relative advantage in fostering their offspring along the social ladder. This is in accordance with sociological studies on social stratification and mass education (Shavit and Blossfeld, 1993). 
Table 4. Effect of increase in the number of university courses on the probability of holding a university degree or being a full-time university student

Effect of expansion

a. By social class

Working class or out of labour force

Petite bourgeoisie

Bourgeoisie

$R$-squared

b. By cultural capital

Low

Medium

High

$R$-squared

Regional control variables

$\Delta$ Unemployment rate (1990-2000)

Growth rate per capita real disposable income (1990-2000)

$\Delta$ Secondary school graduates (1990-2000)

No. observations
(1)

(2)

(3)

(4)

(5)

$\begin{array}{llll} & 4,822 & 4,822 & 4,822\end{array}$

Notes: The treatment is the average yearly increase in the number of university courses in the period 1990-2000 at regional level divided by the population aged 19 in 1991. Absolute value robust $z$ statistics in brackets (standard errors are clustered at region by cohort level); * significant at 10 per cent; ** significant at 5 per cent; *** significant at 1 per cent. Models also include cohort of birth dummies, age, region of residence dummies, gender, parental social class, parental cultural capital, and the interaction between parental social class and the post-reform dummy (section a) or the interaction between cultural capital and the post-reform dummy (section b). Observations are weighted to population proportions.

The effect of the creation of new courses on the probability of being a full-time student or having a degree is reported in Table 4. In this case, HE expansion seems to have had an effect only on middle-class individuals, amounting to about +7 percentage points in the specification in column (5). The increase in HE supply also had a positive effect on individuals with medium levels of cultural 
Table 5. Effect of increase in the number of university locations on the probability of holding a university degree or being a full-time university student

\section{Effect of expansion}

a. By social class

Working class or out of labour force

Petite bourgeoisie

Bourgeoisie

$R$-squared

b. By cultural capital

Low

Medium

High

$R$-squared

Regional control variables

$\Delta$ Unemployment rate (1990-2000)

Growth rate per capita real disposable income (1990-2000)

$\Delta$ Secondary school graduates (1990-2000)

No. observations

(1)

(2)

(3)

(4)

(5)

$\begin{array}{ccccc}0.163 & 0.290 & -0.200 & 0.163 & 0.465 \\ {[0.08]} & {[0.15]} & {[0.10]} & {[0.08]} & {[0.24]} \\ 2.585 & 2.741 & 2.309 & 2.585 & 2.920 \\ {[1.31]} & {[1.51]} & {[1.19]} & {[1.31]} & {[1.60]} \\ -5.528 & -5.865 & -5.539 & -5.528 & -5.529 \\ {[1.27]} & {[1.35]} & {[1.23]} & {[1.27]} & {[1.23]} \\ 0.24 & 0.24 & 0.24 & 0.24 & 0.24\end{array}$

$\begin{array}{ccccc}0.497 & 0.591 & 0.139 & 0.497 & 0.721 \\ {[0.27]} & {[0.34]} & {[0.07]} & {[0.27]} & {[0.40]} \\ 1.960 & 1.987 & 1.821 & 1.960 & 2.319 \\ {[0.62]} & {[0.65]} & {[0.61]} & {[0.62]} & {[0.76]} \\ -8.776 & -8.674 & -9.123 & -8.776 & -8.279 \\ {[1.23]} & {[1.21]} & {[1.25]} & {[1.23]} & {[1.15]} \\ 0.24 & 0.24 & 0.24 & 0.24 & 0.24\end{array}$

$\begin{array}{ccccc}\text { No } & \text { Yes } & \text { No } & \text { No } & \text { Yes } \\ \text { No } & \text { No } & \text { Yes } & \text { No } & \text { Yes } \\ \text { No } & \text { No } & \text { No } & \text { Yes } & \text { Yes } \\ 4,822 & 4,822 & 4,822 & 4,822 & 4,822\end{array}$

Notes: The treatment is the average yearly increase in the number of university sites in the period 1990-2000 at regional level divided by the population aged 19 in 1991. Absolute value robust $z$ statistics in brackets (standard errors are clustered at region by cohort level). Models also include cohort of birth dummies, age, region of residence dummies, gender, parental social class, parental cultural capital, and the interaction between parental social class and the post-reform dummy (section a) or the interaction between cultural capital and the post-reform dummy (section b). Observations are weighted to population proportions.

capital and a negative effect on individuals with high levels of cultural capital [about +14 and -25 percentage points, respectively, column (5)]. In this case too the high and negative effect for highcultural capital individuals may be driven partly by endogenous migration. Table 5 shows no statistically significant effect of the creation of new sites on the educational outcome considered. 
Overall, the results in Tables 2 and 4 suggest that the creation of new courses might have had a beneficial effect on enrolment rates more than on graduation rates when considering the age group 23-31 in the period under study. Indeed, the rise in enrolments among young students might not have translated immediately into an increase of the number of young graduates due to the increase in time to graduation. Put another way, HE supply might have benefited 'marginal' students in particular (i.e. the poorest ones in terms of economic or cultural resources), who may also be relatively more likely to graduate late (fuori corso). As to the insignificant correlations generally found for the creation of new sites at regional level, it must be noted that the time and cross-region variation in this variable is much smaller than that in the creation of new courses, and this might have somewhat affected statistical significance. ${ }^{23}$

\subsection{Robustness checks}

As we have anticipated, our analysis is subject to some qualifications. As our data do not record the region of residence at age 19, when the decision to enrol in HE is typically made, this introduces a potential problem of endogenous mobility. So far, we are currently making the assumption that the current region of residence is also the region where individuals resided at age 19. However, this is not necessarily the case. Individuals may have changed residence between age 19 and the age at which they are observed in our analysis. In particular, some individuals may have moved to regions in which the expansion of HE supply was greater because they wanted to enrol in HE or because these regions offered more employment opportunities or better marriage opportunities (see Currie and Moretti, 2003). In this case, we would be likely to overestimate the effect of HE supply due to positive self-selection. In this section, we try to address this problem by replicating the baseline specification on the sample of individuals for whom the current region of residence coincides with the region of birth. Although some individuals might have gone to study in a different region from the one in which they were born and then returned to their region of birth after completing their studies, ${ }^{24}$ we claim that for this sample the likelihood of the region where they resided at age 19 coinciding with the current residence (i.e. they are stayers) is high, given the low geographical mobility in Italy. We limit the robustness checks in this section to the regressions using the creation of new courses as a measure of $\mathrm{HE}$ expansion and analysing 
the likelihood of being a full-time university student or holding a degree, which gave the most significant and robust results.

Column (1) of Table 6 reports the results of this first robustness check for the specification including all regional control variables. This specification must therefore be compared with that reported in column (5) of Table 4. Results are broadly consistent. However, focusing on the sample of stayers produces a remarkable increase in the absolute value of the negative effect of creation of new courses on individuals with high cultural capital. We anticipated in the introduction to Section 3 that endogenous migration is likely to bias our estimates especially for high-background individuals, for whom migrating to study is an option. Indeed, when focusing on highbackground stayers the problem of negative selection with respect to enrolment in HE is likely to be exacerbated, because the fraction of those who did not study among the stayers is probably higher compared with low-background individuals. One speculation that cannot be explored further with SHIW data but that deserves further investigation with richer data sets is that high-background individuals react to the local increase of $\mathrm{HE}$ by moving to older and well-established institutions perhaps located in other regions in order to maintain their social advantage over low-background individuals and to avoid mixing with them.

In order to investigate further the issue of endogenous mobility we run another robustness check. We also consider individuals aged $35-43$ in the 2002 wave and interact supply expansion with the two age groups, 23-31 and 35-43, respectively. Individuals aged 35 in 2002 were 23 in 1990, whereas those aged 43 in 2002 were 31 in 1990, hence HE expansion should have not affected their educational attainment as they were more likely to have completed university or entered the labour market well before the 1990s. A strong positive correlation between HE expansion and their tertiary educational achievement would instead cast serious doubts on our identifying assumption, which uses current residence as a proxy of residence at age 19, when enrolment decisions are typically made. Column (2) of Table 6 shows that this is not the case. Indeed, the results for the younger cohort (aged 23-31) are qualitatively similar to those reported in Table 4 column (5), whereas the interaction terms between the increase in supply and the older age group (35-43) turn out to be either insignificant or significant and negative. ${ }^{25}$ The only interesting difference is that now the positive effect on low-cultural capital individuals also becomes statistically significant, although amounting to less than one-third of that of individuals with medium 
Table 6. Robustness checks on the effect of creation of new courses

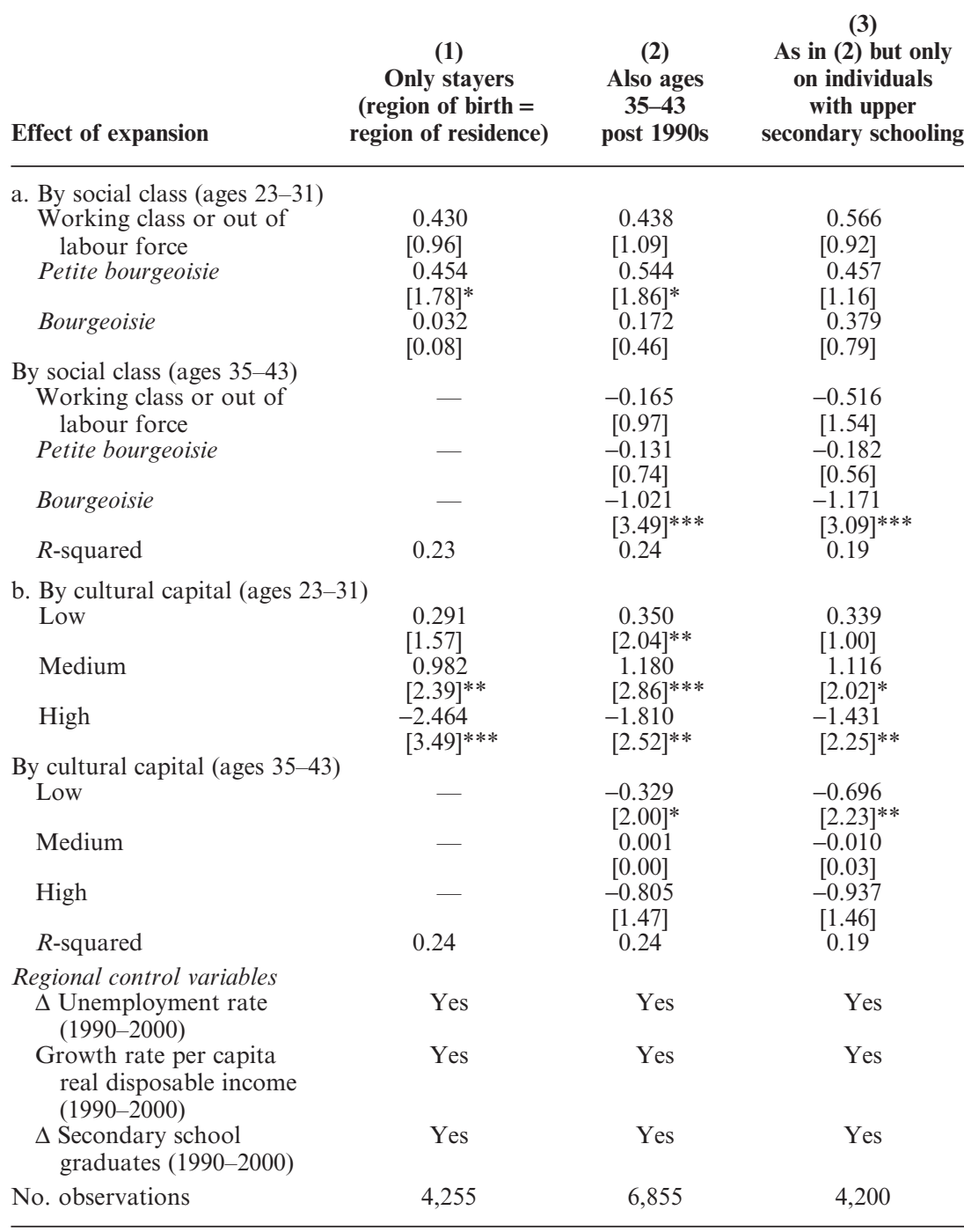

Notes: The treatment is the average yearly increase in the number of courses in the period 1990-2000 at regional level divided by the population aged 19 in 1991. Robust $z$ statistics in brackets (standard errors are clustered at region by cohort level); ${ }^{*}$ significant at 10 per cent; ${ }^{* *}$ significant at 5 per cent; *** significant at 1 per cent. Models also include cohort of birth dummies, age, region of residence dummies, gender, parental social class, parental cultural capital, and the interaction between parental social class and the post-reform dummy (section a) or the interaction between cultural capital and the post-reform dummy (section b). Observations are weighted to population proportions. 
levels of cultural capital (about +5 and +15 percentage points, respectively). This suggests that the positive effect of HE supply on low-cultural capital individuals shown in Table 4 may not have been very precisely estimated due to the small sample size.

Last but not least, we investigate how our results change when we restrict the sample only to upper secondary school graduates. The estimates are reported in Table 6 column (3). In order not to reduce the sample size too much, we also include in the estimation sample individuals in the 35-43 age group (in the post-reform period) as in the previous estimation. Column (3) shows that results are highly consistent in magnitude and sign with those of the previous column, although the coefficients are more imprecisely estimated.

\subsection{Interpretation of results}

Why did the expansion of educational supply increase access to HE by low- and medium-background individuals (social class or cultural capital) but not their chances of obtaining a degree before age 31 ? Several hypotheses can be put forward. ${ }^{26}$

One explanation relates to what is known as the 'parking lot' hypothesis. ${ }^{27}$ As emphasized by Dornbusch and Giavazzi (2000), during the 1990s many young Italians in their 20s were simply hanging around on campus; in other words they were simply waiting for a decent job offer, which might take some time, before dropping out from university. In this respect, the concomitant poor labour market performance was also crucial. Indeed, in such a situation having student status (even without making any effort to study) was considered by students as a way of 'fooling' both employers and their families and not being viewed as unemployed workers. The local supply expansion of the 1990s might have contributed to this phenomenon by making the cost of enrolling and staying longer in HE cheaper for the least academically able and motivated students, therefore increasing the likelihood of enrolment and reducing that of an early dropout. Here, it is important to note that the 'parking lot' hypothesis is relevant for the explanation of our findings only if HE expansion reduced 'late' dropout, i.e. the dropout of relatively older students. As we are considering full-time students, older students are also likely to be 'slower' in their study careers. ${ }^{28}$ Indeed, it is only in this case that we would find a positive correlation between $\mathrm{HE}$ expansion and the status of full-time student at relatively older ages (such as 23-31). ${ }^{29}$ Unfortunately, data to examine 'late' student dropout are not readily available. ${ }^{30}$ 
Table 7. Evidence from the Italian 'Survey of graduates' (ISTAT)

\begin{tabular}{|c|c|c|c|}
\hline \multirow[b]{2}{*}{ Descriptive statistics } & \multicolumn{3}{|c|}{ Cohorts of graduates } \\
\hline & 1992 & 1995 & 1998 \\
\hline \multicolumn{4}{|l|}{ Students' employment } \\
\hline Per cent continuous & 31.42 & 11.41 & 13.30 \\
\hline Per cent sporadic or seasonal ${ }^{\mathrm{a}}$ & & 45.00 & 45.22 \\
\hline Per cent never worked & 68.58 & 43.59 & 41.48 \\
\hline \multicolumn{4}{|l|}{ Students' graduation times } \\
\hline Per cent legal duration & 15.87 & 11.85 & 10.08 \\
\hline Per cent $\leq 2$ years fuori corso & 48.83 & 42.53 & 47.17 \\
\hline Per cent $>2$ years fuori corso & 35.30 & 45.62 & 42.74 \\
\hline
\end{tabular}

Notes: Our computations on the ISTAT 'Survey of graduates' for the graduate cohorts 1992, 1995, and 1998.

a For the 1992 cohort the information on the type of employment is not available.

A second explanation may be that by increasing the access of individuals from poorer (economically or culturally) family backgrounds, HE expansion also increased the fraction of students who have to work to finance their studies and who also generally have longer graduation times. The joint consideration of cost reductions (due to the possibility of enrolling at the local university) and longer duration could have both reduced the incentive to drop out for working students and had negative effects on graduation times. Indeed, although in ISTAT's 'Surveys of university graduates' we observe both an increase in the percentage of working students and an increase in the percentage of graduates fuori corso during the 1990s (Table 7), in the 'Surveys of secondary school diplomaholders' for 1995 and 1998 we observe a small reduction in student dropout within 3 years from secondary school completion (from 14.5 per cent to 13.9 per cent, respectively).$^{31}$ A related explanation is that the increasing HE supply could have partly relaxed credit constraints for individuals from low backgrounds, thus increasing their likelihood of participating in $\mathrm{HE}$, but that these individuals lacked the cultural resources and competencies needed to progress in HE. The explanation whereby the HE educational achievement of lowbackground individuals is not really limited by short-term credit constraints but by long-term factors is gaining a certain popularity in the USA (see, for instance, Cameron and Heckman, 2001; Cameron and Taber, 2004). This may also be relevant for Italy, where there is 
a strong socio-economic gradient already in secondary school achievement and in adolescents' literacy levels (see Bratti et al., 2007; Checchi and Flabbi, 2007) and therefore low-background and highbackground individuals with a secondary school diploma may differ considerably in terms of their levels of knowledge and competencies, which are then necessary to succeed in HE. Hence, policies aimed at increasing equality in $\mathrm{HE}$ achievement among the population should be targeted at the primary and secondary school levels and not only specifically at tertiary level.

A final possible rationalization comes from the supply side. Indeed, it is widely recognized that neither the teaching system nor the funding system provides Italian HE institutions with an incentive to be efficient, where 'efficiency' is broadly defined as the rate and/or the speed at which students are 'transformed' into graduates. As far as the teaching system is concerned, an Italian student can remain in such a situation lifelong: students are allowed to repeat exams without any limitation (regardless of whether they fail the exam or are not satisfied with the marks obtained); in addition they can renew enrolment for an unlimited number of years, even if they did not pass any exam in the previous year: as a consequence they have no limitation as to graduation time. All these factors certainly do not contribute to speeding up students' university careers. As to the funding system, most HE funding in the period under study came from the central government and more than 90 per cent of the funds were allocated on an historical basis (Perotti, 2002). Therefore, the funding formula did not provide HE institutions with any incentive to be efficient. This was so widely acknowledged that the study of a new funding formula was undertaken by the Italian National Committee for Evaluation of the University System (CNVSU) in 2004 (CNVSU, 2005). The new formula attempted to tie a large part of the allocation of public funds to the outcomes of the educational process measured in terms of the speed of students' careers (crediti formativi, i.e. student credits) and the number of graduates in each institution (although penalizing institutions with high percentages of fuori corso). Given the lack of efficiency of the Italian university system, it may be that universities were not able, or willing, to manage their suddenly achieved autonomy efficiently by speeding up the career of their students. ${ }^{32}$ Indeed, as we have seen in Section 2, autonomy was mainly exploited for reasons unrelated to students' needs but mostly connected with academic and local interests, probably leading to a further reduction in the efficiency of HE institutions. To the best of 
our knowledge no study has seriously tested this hypothesis by measuring the trend of universities' efficiency during the 1990s. Curiously, however, aggregate figures (MIUR, 2006) point to a constant increase in the fraction of students fuori corso during the years of HE expansion (from 32.5 per cent in the academic year $1994 / 95$ to 42.4 per cent in the academic year 1999/2000). ${ }^{33}$

\section{Concluding remarks}

The changes in the regulation of HE introduced in Italy during the 1990s prompted a spectacular increase in supply, which included both a wider range of degrees and many new sites.

We use data from the Bank of Italy's SHIW to investigate the effect of the expansion of HE supply at regional level on two distinct educational outcomes: (1) the likelihood of holding a university degree; and (2) the likelihood of holding a degree or of being a full-time university student.

As the expansion of supply might have benefited individuals with different family backgrounds differently, we allow for heterogeneity of its effect across individuals.

We do not find any strong evidence of an effect of HE expansion on the likelihood of graduation, either when expansion is measured in terms of new courses created or when it is measured in terms of new sites opened.

In contrast, we find robust evidence of a positive effect of $\mathrm{HE}$ expansion on student enrolment and retention only when it is measured in terms of creation of new courses. When considering the social class of origin, our analysis suggests that individuals from the middle class benefited most from the expansion of the Italian HE system. When considering cultural capital, our estimates suggest that supply expansion benefited mainly individuals with low and medium levels of cultural capital.

Finally, we advance some possible interpretations of the contrast between a positive effect of $\mathrm{HE}$ expansion on increasing access to HE but not on successful completion of HE. Some could be related to the 'parking lot' hypothesis, to an increase in the fraction of working students, or to a reduction in the efficiency of HE institutions. However, further analysis and better data are needed to distinguish between these hypotheses.

Although the limitations of the data mean that the analysis in this paper should not be considered conclusive evidence on the 
ineffectiveness, in terms of reduction of inequality of HE attainment, of educational policies based on the expansion of supply, it is nonetheless indicative of a potential problem. Indeed, our overall results suggest that the rapid expansion of HE supply in the 1990s may have only produced a limited increase in equality of opportunity in terms of completion of tertiary education and partly explain why tertiary educational attainment in Italy is still strongly related to parents' education (Checchi et al., 2007).

\section{Appendix A}

\section{A snapshot of the university system in Italy}

The Italian university system has traditionally been organized centrally. Even the oldest universities (Turin, Pavia, Genoa, Cagliari, and Sassari) were instituted by royal decree in 1859 , or were approved by pre-unitary states (Pisa, Siena, Bologna, Parma, Modena, and Macerata) and then incorporated in the dawning Italian state. This was reaffirmed by the Fascist regime in 1933 with a unified framework law (Testo unico, regio decreto $n .1952$ $31 / 8 / 1933$ ) that classified state (fully financed) and free (partially subsidized) universities. Given the stationary number of enrolments, the list of state universities remained largely unaltered until the reform of 1969, when university admission was opened to all students who had completed 5 years of secondary education (before that, graduates from technical or professional secondary school did not have full right to enrol in HE: they could only enrol in university courses related to their secondary-school field - for instance, secondary school diploma-holders in accounting could only enrol in economics).

The rising pressure of applications pushed the Italian universities towards a mass university system, which in turn forced the legislator to approve the opening of new universities. These included: Udine in 1978; the 2nd university of Rome, Viterbo, and Cassino in 1979; Potenza in 1981; l'Aquila, Chieti-Pescara, Brescia, Campobasso, Reggio Calabria, Verona, and Trento in 1982 (transformation of pre-existing HE institutions).

A substantial reform of the functioning of the universities (in terms of management, hiring, teaching loads) came in 1980 (Law n. 382 11/7/1980), which provided that any variation in the existing university supply should be included in a development plan (piani 
triennali), to be approved by the Minister of Education every 3 years (Law n. 590 14/8/1982). However, any opening of new universities required a specific law to be passed by Parliament.

It was a decade later that the requirement of parliamentary approval was abandoned (Law n. 341 19/12/1990), whereas the inclusion in a development plan was still retained. However, universities gained autonomy to advance proposals for new initiatives to the Ministry. Up to that point, a university professor was appointed to a chair corresponding to a specific course and in order to introduce a specific degree a university had to fulfil the requirement of a specific law listing the number and names of courses needed to attain the degree. This was also the rationale for the legal value of a degree across the country: as the teaching was in principle identical across the country, there was no reason to presume that identical degrees obtained in different universities could be different, given a centrally organized hiring procedure through national competitions. Thus, in order to offer a new degree (to be selected from a closed list of admissible degrees) a university needed an almost complete new faculty corresponding to the courses to be taught.

Since 1990 the system has been made more flexible. University professors are associated to research fields (settori scientificodisciplinari) and not to teachings, thus relaxing the resource constraint. Universities are entitled to propose new degrees, although formal approval of the central government is still needed. A further degree of flexibility was introduced in 1990 but implemented only in 1995 , i.e. the possibility to create shorter courses, 2-3 years long, called diplomi universitari. This was in anticipation of the fully fledged reform in accordance with the 'Bologna process', introduced in 1999 and commonly known as the ' $3+2$ ' reform, as it reorganized the teaching as a 3 -year bachelor degree followed by a 2-year master-equivalent degree (Law n. 509 3/11/1999). This law became operational after 2001.

The central government has retained its initiative by forcing larger universities (those exceeding 40,000 enrolled students) to split into two. The provision was contained in the budget law for 1997 (Law n. 662 23/12/1996) and allowed the creation of the third university of Rome, the second university of Milan, plus new universities such as Teramo, Catanzaro, Benevento, Insubria at Varese, Piemonte Orientale in Vercelli, and Foggia.

The development plans approved for 1991-93 and 1994-96 allowed (and financed) the expansion of HE to cover the entire national territory and balance the allocation of funds with respect 
to the southern regions (with aim of increasing the equality of opportunity to access HE).

In order to move towards a more decentralized system, during the 1990s the Italian Parliament passed a series of laws granting Italian universities a substantial, previously inexperienced, autonomy. A ground-breaking law (Law n. 168 12/5/1989) reformed the centralized organization, creating a Ministry for University and Scientific and Technological Research (Ministero per l'Università e la Ricerca Scientifica e Tecnologica, MURST) as a separate body from the Ministry of Education. This law established the general principles for self-government, giving the universities the right to have their own statutes. As a consequence, universities gained both teaching and financial autonomy. Autonomy meant the freedom to allocate the funding from central government, which remained the prevailing source of funds. Some autonomy in setting student fees was granted with the budget law of 1993 (Law n. 537 24/12/1993), in conjunction with a reduction of funding from the government. Universities were allowed to manage their teaching and research activities, but the creation of new courses was still subject to central approval. In addition, professors' salaries and new hirings were still the preserve of central government. This produced a 'soft budget constraint' as the bulk of the financing needs remained covered by central government (from the universities' point of view, expanding the supply of courses was also a way of applying additional pressure on the government to obtain new professors). The process of progressive devolution was completed in 1998, when universities were allowed to open (or close) new schools (facoltà) and/or courses without central approval, conditional on self-financing of the initiative (DPR n. $2527 / 1 / 1998$, issued in application of a general trend to devolution required by Law n. 59 15/3/1997, also known as legge Bassanini). Although an overall evaluation of this decade remains to be written, it is well summarized by the words of a former deputy minister of Higher Education in the period 1996-2001: 'If necessary, the final assessment is that universities and research institutes obtained their autonomy in an uncertain framework, which was incomplete from a legislative viewpoint and was managed in a contradictory way' (L. Guerzoni in the introduction to Masia and Santoro, 2006, p. 16 - our translation). 


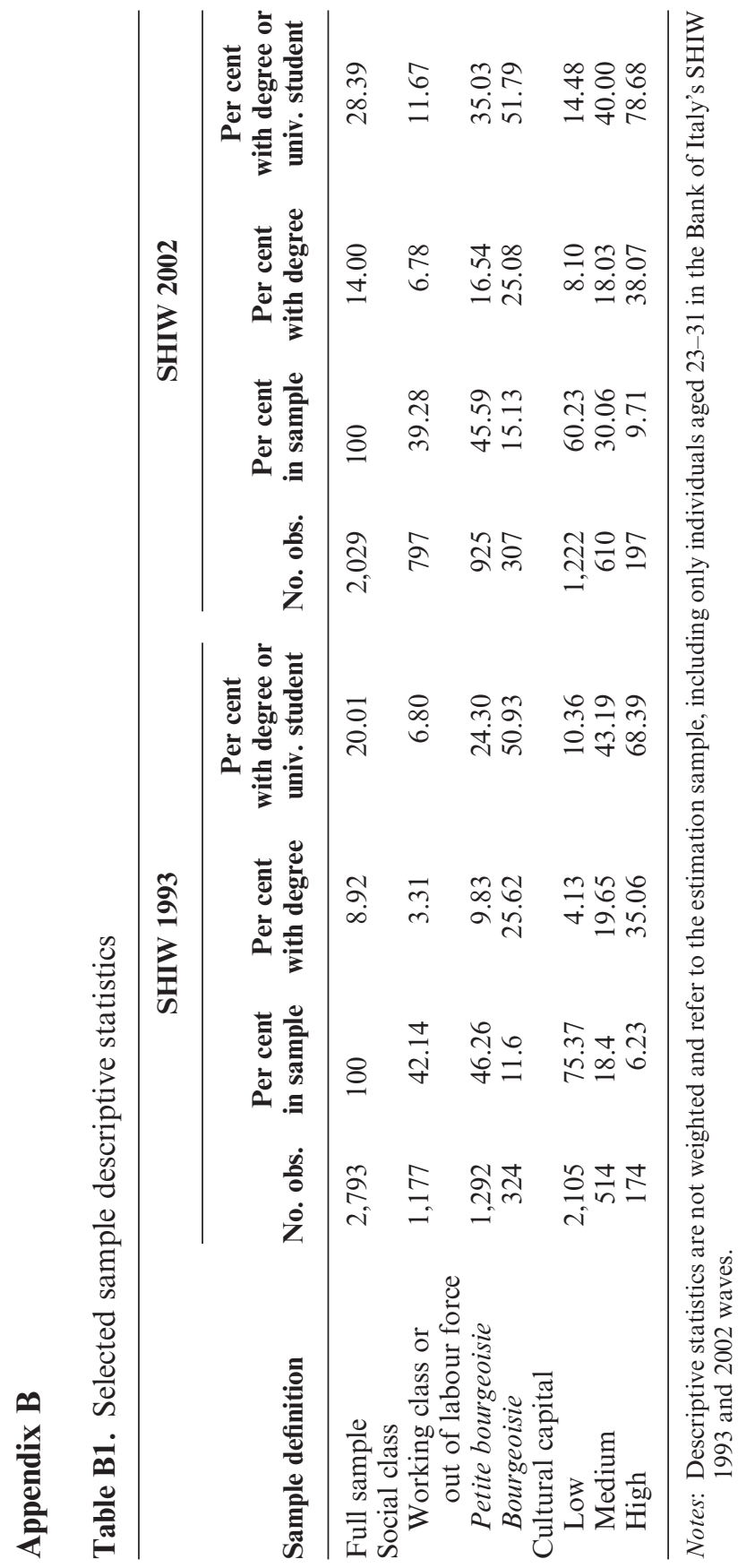




\section{Notes}

${ }^{1}$ Because of the lack of appropriate data, we do not pursue a non-mutually exclusive explanation, typically referred to as 'genetic'. In this view, the correlation between children's and parents' levels of education at least partly reflects intergenerational transmission of ability. In this sense, the fact that children from highly educated (or wealthier) parents are more likely to continue in HE could simply indicate that they have a higher level of ability. However, when proxies for abilities are available (as in Checchi and Flabbi, 2007, who use the OECD Programme for International Student Assessment test scores), it has been shown that in Italy educational decisions at secondary and tertiary level are more affected by family background than by individual ability, especially compared with similar countries, such as Germany. In this paper, we do not have good proxies for unobserved ability as our data set only contains the final marks at the exit of the highest educational attainment (which, because they do not represent the score of a standardized test, are extremely difficult to compare between individuals). Consequently, in what follows we interpret intergenerational correlations as mostly driven by pecuniary and non-pecuniary family background effects (nurture) rather than by nature (i.e. the genetic endowment of ability). We nonetheless include a robustness check in Section 3.2, in which we investigate the effect of restricting the analysis only to individuals who have completed upper secondary schooling.

${ }^{2}$ In most cases local councils sponsored projects to open university branches in their cities because of the prestige of having HE courses taught locally (and because of the approval of the families due to the lower costs of attendance).

${ }^{3}$ Opening a new school from scratch was difficult. In the vast majority of cases, a group of professors working in the same (or related) fields asked for a new school to be created there or elsewhere. For instance, the School of Economics of the University of Siena, which has traditionally offered three types of degree - economics, statistics, and banking - increased its supply to eight courses. At the end of the 1990s the school was able to offer six more degrees: economics of financial markets, environmental economics, public economics, business administration, economics of small and medium enterprises (in a different location, Arezzo), and economics and sustainable development (in another location, Grosseto).

${ }^{4}$ Shorter (2- and 3-year) degrees (corsi di diploma universitario) were also introduced: in 2000 there were 956 of them. The vast majority of students, however, continued to enrol in corsi di laurea.

${ }^{5}$ Because the number of individuals living in the Valle d'Aosta region is small, they are assigned the values of Piedmont (the only bordering region) for both the expansion of supply and the regional controls.

${ }^{6}$ Rostan (2006) reports this figure to be 86 per cent (p. 207). Therefore, the fraction will also be higher when measured at regional level. In the 2002 SHIW, which provides data on the HE institutions in which individuals studied, 84 per cent of the graduates aged 23-31 were resident in the region in which they studied, 79 per cent studied in the region in which they were born, and for three-fourths of the sample (78 per cent) regions of study, residence, and birth coincide. These figures are slightly lower for Southern Italy ( 76 per cent, 80 per cent, and 75 per cent, respectively). 
${ }^{7}$ This problem is probably more serious for individuals from wealthy families, who can afford the cost of studying in a different region, and those residing in the South, who are more likely to move elsewhere for study reasons than students from the Centre and North of Italy.

${ }^{8}$ A similar positive correlation would still be of interest as it would provide a measure of attractiveness of a certain region to highly skilled labour, but it would not capture the causal effect of HE supply on the educational attainment of the resident population, which we are interested in.

${ }^{9}$ In what follows we sometimes use the expression 'probability of being a student' to mean the 'probability of being a full-time student'. The focus on full-time students is related to the nature of SHIW data, which do not identify part-time students.

${ }^{10}$ This indicator could be improved either by an increase in enrolments at invariant dropout rates or by a reduction in dropout rates at invariant enrolment, or even by a simultaneous increase in enrolment and reduction in dropout. Our data do not report dropout information and therefore we cannot distinguish between these potential effects.

${ }^{11}$ This could be an intended result of the reform. For instance, some of the newly created courses (called diploma universitari — see Appendix A) had a shorter duration ( 3 instead of 4 or 5 years).

${ }^{12}$ In the academic year 2001-2 the Italian system of HE was changed again with the introduction of the ' $3+2$ ' system, which envisaged 3-year first level degrees followed by 2-year second level degrees (generally known as the 'Bologna process'). In this paper we only consider the effect of the expansion of university supply during the 1990 s, i.e. before the implementation of the 'Bologna process'. For a study focusing on the ' $3+2$ ' reform see Bondonio (2006). As we are considering the 2002 wave of the SHIW, the second measure of educational attainment, which also considers full-time students, may be affected by the ' $3+2$ ' reform: some students might have decided to enrol in HE in 2001 after the introduction of the reform. We do not expect this to affect our analysis as we consider individuals over 23 years of age who self-declared to be full-time students. Most students who decided to go back to university after the ' $3+2$ ' reform were likely to be working full-time or part-time, whereas we expect only few of them to have converted into full-time university students, withdrawing from the labour market. However, we also run a robustness check of all regressions reported in this paper by excluding full-time university students with past working experience from the analysis (39 individuals in our estimation sample), without any change in the results.

${ }^{13}$ In particular, individuals who were 31 in 2002 were 19 in 1990, whereas those who were 23 in 2002 were 19 in 1998. It is perhaps important to note that there is another potential problem with the baseline experiment described, namely a possible time overlap of 'untreated individuals' with the time span of the reform. Indeed, nothing prevents the sample taken from the 1993 wave from including individuals who might have benefited in part from the reform. As for the likelihood of holding a degree, we know that individuals who possessed a university degree in 1993 must have enrolled at university before 1990. However, some students could have enrolled in the new courses created after 1990, as this possibility was allowed by law. The problem may become more serious when we consider our second outcome variable, the likelihood of being a university student or obtaining a degree. Indeed, this variable can reflect late enrolments occurring after 1990. In a previous version of the paper, in which we estimated a common effect of HE 
expansion without interacting it with individual family background, we considered the 1991 (less subject to the overlap problem) and the 2002 cohorts, obtaining very similar results to the present ones. We take this as an indication that partial overlapping is less of a problem.

${ }^{14}$ For a similar framework see Duflo (2001).

${ }^{15}$ In the regressions we do not control for the fact of possessing an upper secondary school diploma and do not restrict the sample to individuals with an upper secondary school diploma. Possessing an upper secondary school diploma can be considered an endogenous outcome that is also affected by parental characteristics (such as education) and for this reason we prefer to exclude it. Moreover, in the regressions: (1) we already control for household background that, as we have said, is a strong predictor of an individual's education in Italy; and (2) we consider the effect of including the increase in the number of upper secondary school graduates at regional level, which may be correlated with the expansion of HE supply (see column (4) of Tables 2-5). The consequences of restricting the sample to individuals with an upper secondary school diploma (those who are entitled to enter HE) are investigated in Section 3.2.

${ }^{16}$ In the SHIW family background controls are collected from two separate sources. For individuals who are living with their parents, family background variables can be obtained from the main questionnaire as it routinely collects educational achievements and job qualifications of all family members. For individuals living away from their parents (or for those whose parents are not co-habiting because of divorce/migration/death), family background variables have to be obtained from the intergenerational-information section (regarding the time when the parents were the same age as the individuals), which was included starting from the 1993 wave. As Francesconi and Nicoletti (2006) show, it is important to keep both co-residents and non-co-residents in the estimation sample, as co-residence may not be random with respect to our outcome of interest.

${ }^{17}$ However, in the regressions we do control for individual level variables that may proxy liquidity constraints or income effects (namely parental social classes and educational levels).

${ }^{18}$ Although the LPM delivers unbiased and consistent estimates when variables uncorrelated with the included covariates are omitted from the regression, nonlinear models such as probit or logit models do not have this property (see, for instance, Cramer, 2005).

${ }^{19}$ The implication for the econometric analysis is that the corresponding 'negative' (zero) outcomes in the linear probability models are the probability of never having enrolled in $\mathrm{HE}$, or of having enrolled and then dropped out from $\mathrm{HE}$, or of being a full-time or part-time student when considering the first indicator, and the likelihood of never having enrolled in $\mathrm{HE}$, or of having enrolled and dropped out from $\mathrm{HE}$ or of being a part-time student when considering the second indicator, respectively.

${ }^{20}$ As family background is measured in terms of categories, each one of the family background categories is represented by one dummy variable BACKGROUND. We estimate two separate specifications regarding as BACKGROUND either family education or social class. The dimension of family background that is not interacted with HE supply is then included in $\mathrm{X}$.

${ }^{21}$ The fact that estimates of the effect of supply cease to be statistically significant when change in per capita income is included suggests that finding an insignificant effect is not mainly determined by the small sample size. 
${ }^{22}$ This might look quite high. However, ceteris paribus it would be the effect produced on high-background individuals only by HE expansion. Their actual enrolment or graduation rates will then be determined by the other characteristics they possess as well (such as possible changes in the return to family background, $\mathrm{b}_{4}$ in equation [1]).

${ }^{23}$ For this reason we plan to exploit provincial variation in the creation of new sites by matching HE supply data with Italian Census data (ISTAT) to explore this hypothesis further. Although Census data offer the opportunity of having larger estimation samples, data on parental background is not reported for individuals who are not living with their parents.

${ }^{24}$ However, this should lead to an underestimation of the effect of HE supply.

${ }^{25}$ A similar finding is reported in Currie and Moretti (2003, p. 1515).

${ }^{26}$ Unfortunately, SHIW data do not enable us to disentangle their relative importance. Therefore, whenever possible we use alternative data sources to provide some indirect evidence on the validity of any of the proposed interpretations.

${ }^{27}$ For a recent economic formalization of this hypothesis see Becker (2006).

${ }^{28}$ Di Pietro (2006) finds that labour market dynamics are important for first-year university dropout. Indeed, his study shows a strong negative correlation between first-year university dropout rates and unemployment rates at regional level, i.e. students are more likely to continue in their studies when the labour market does not offer them good employment opportunities. However, his study does not consider the effect of labour market conditions on students' 'late' dropout or graduation rates.

${ }^{29}$ An alternative explanation could be that HE expansion increased enrolment of mature students. However, this is probably irrelevant for our analysis as mature students are very unlikely to self-define as full-time students and therefore to be recorded as students in the SHIW.

${ }^{30}$ Indeed, a natural source of data for testing this hypothesis would be the 'Survey of secondary school diploma-holders' run by ISTAT. At present there are only three waves of this survey, for the cohorts graduating in 1995, 1998, and 2001, respectively. However, from this survey it is only possible to study dropout rates after 3 years since course initiation (approximately age 22), which is not useful for our purposes.

${ }^{31}$ Computing dropout rates on the 'Survey of secondary school diplomaholders' is not straightforward. We used questions 16 and 17 for the 1995 cohort and questions 18, 19, and 34 for the 1998 cohort, including all observations with non-missing answers. It must also be noted that dropout rates are considerably lower than those recorded in the universities' administrative data. This may due to several reasons, among which the fact that administrative data also take into account changes of courses or that in survey data early dropout students may declare they never enrolled in HE (cf. Cingano and Cipollone, 2007).

${ }^{32}$ One should not forget that students fuori corso (namely students that remain enrolled for a period exceeding the prescribed course length) are a source of funds for the universities as they pay tuition for each enrolment year, without attending courses or participating in campus life.

${ }^{33}$ This rise in the phenomenon of fuori corso should by no means be considered direct proof of a reduction in the efficiency of $\mathrm{HE}$ institutions as it may also be due to a worsening of student intake (i.e. a demand-side explanation), which points to our first two explanations. 


\section{References}

Becker S. (2006) 'Introducing Time-to-educate in a Job Search Model', Bulletin of Economic Research 58: 61-72.

Besley T. and Case A. (2000) 'Unnatural Experiments? Estimating the Incidence of Endogenous Policies', Economic Journal 110: F672-F694.

Bondonio D. (2006) 'La Valutazione dell'Impatto Netto della Riforma' in Bini M., Bondonio D. and Crocetta C. (eds.) Gli Effetti della Nuova Riforma dei Cicli e degli Ordinamenti Didattici, Research Report to Ministero dell'Istruzione, dell’Università e della Ricerca, Comitato Nazionale per la valutazione del sistema universitario.

Borland J., Yi-Ping T. and Wilkins R. (2005) 'Experimental and Quasiexperimental Methods of Microeconomic Program and Policy Evaluation', Melbourne Institute Working Paper Series, Working Paper No. 8/05, Melbourne: The University of Melbourne.

Bratti M., Checchi D. and Filippin A. (2007) 'Geographical Differences in Italian Students' Mathematical Competencies: Evidence from PISA 2003', Giornale degli Economisti e Annali di Economia 66: 299-333.

Cameron S. and Heckman J. (2001) 'The Dynamics of Educational Attainment for Black, Hispanic, and White Males', Journal of Political Economy 109: 455499.

Cameron S. and Taber C. (2004) 'Estimation of Educational Borrowing Constraints Using Returns to Schooling', Journal of Political Economy 112: 132-182.

Carneiro P. and Heckman J. (2003) 'Human Capital Policy', NBER Working Paper No. 9495, Cambridge, MA: National Bureau of Economic Research.

Checchi D. (2003) 'The Italian Educational System: Family Background and Social Stratification' in ISAE (ed.) Annual Report on Monitoring Italy, Rome: ISAE: 131-176.

Checchi D. and Flabbi L. (2007) 'Intergenerational Mobility and Schooling Decisions in Germany and Italy: The Impact of Secondary School Tracks', IZA DP No. 2876, Bonn: IZA.

Checchi D., Fiorio C. and Leonardi M. (2007) 'Intergenerational Persistence in Educational Attainment in Italy', Mimeo, University of Milan, Milan.

Cingano F. and Cipollone A. (2007) 'University Drop-out - The Case of Italy', Bank of Italy Temi di Discussione Working Paper No. 626, Rome: Bank of Italy.

CNVSU (2005) Il modello per la Ripartizione del Fondo di Finanziamento Ordinario (FFO) all'Interno del Sistema Universitario: Riflessioni a Valle dell'Applicazione Sperimentale Prevista dal DM 28 Luglio 2004, doc. 04/05, Rome: Ministero dell'Istruzione, dell'Università e della Ricerca.

Cramer J. (2005) 'Omitted Variables and Misspecified Disturbances in the Logit Model', Tinbergen Institute Discussion Paper 05-084/4, Amsterdam: Tinbergen Institute.

Currie J. and Moretti E. (2003) 'Mother's Education and the Intergenerational Transmission of Human Capital: Evidence from College Openings', Quarterly Journal of Economics 118: 1495-1532.

Di Pietro G. (2006) 'Regional Labour Market Conditions and University Dropout Rates: Evidence from Italy', Regional Studies 40: 617-630. 
Dornbusch R. and Giavazzi F. (2000) 'Italy Must Abandon its University Populism', International Herald Tribune, 14 September.

Duflo E. (2001) 'Schooling and Labor Market Consequences of School Construction in Indonesia: Evidence from an Unusual Policy Experiment', American Economic Review 91: 795-813.

Fernandez R. and Gali J. (1999) 'To Each According to . . . ? Markets, Tournaments and the Matching Problem with Borrowing Constraints', Review of Economic Studies 66: 799-824.

Francesconi M. and Nicoletti C. (2006) 'Intergenerational Mobility and Sample Selection in Short Panels', Journal of Applied Econometrics 21: 1265-1293.

ISTAT (1995) Sistema Educativo e Mercato del Lavoro nel Contesto Internazionale, Rome: Istituto Nazionale di Statistica.

Machin S. and Blanden J. (2004) 'Educational Inequality and the Expansion of UK Higher Education', Scottish Journal of Political Economy 51: 230-249.

Masia A. and Santoro I. (2006) 'L'Università nella XII e XIII Legislatura' (15/4/ 1994-29/5/2001), Universitas Quaderni 20, Associazione RUI.

MIUR (1997) Verifica dei Piani di Sviluppo dell'Università 1986-90 e 1991-93, doc. 4/97, August 1997, Rome: Ministero dell'Istruzione, dell’Università e della Ricerca.

MIUR (2003) Quarto Rapporto sullo Stato del Sistema Universitario, Comitato Nazionale per la Valutazione del Sistema Universitario, Rome: Ministero dell'Istruzione, dell'Università e della Ricerca.

MIUR (2006) L'università in Cifre 2005, Rome: Ministero dell'Istruzione, dell'Università e della Ricerca.

Perotti R. (2002) 'The Italian University System: Rules vs. Incentives', Mimeo, European University Institute, Florence.

Roemer J. (1998) Equality of Opportunity, Cambridge, MA: Harvard University Press.

Rostan M. (2006) Laureati Italiani ed Europei a Confronto. Istruzione Superiore e Lavoro alle Soglie di un Periodo di riforme, Milan: Edizioni Universitarie di Lettere Economia Diritto.

Shavit Y. and Blossfeld H.-P. (eds.) (1993) Persistent Inequality. Changing Educational Attainment in Thirteen Countries, Boulder: Westview Press.

Shavit Y., Arum R. and Gamoran A. (eds.) (2007) Stratification in Higher Education: A Comparative Study, Palo Alto: Stanford University Press. 